\title{
Heating, Efficacy and Dose of Local Hyperthermia
}

\author{
Oliver Szasz, Andras Szasz \\ Department of Biotechnics, St. Istvan University, Paty, Hungary \\ Email: biotech@gek.szie.hu
}

Received 26 November 2015; accepted 17 January 2016; published 20 January 2016

Copyright (C) 2016 by authors and Scientific Research Publishing Inc.

This work is licensed under the Creative Commons Attribution International License (CC BY). http://creativecommons.org/licenses/by/4.0/

c) (i) Open Access

\begin{abstract}
Hyperthermia in oncology needs a definite dose which fixes well the clinical protocols. The temperature is far not a dose, it is mass independent. The half of the mass has the same temperature in equilibrium, so the basic criteria of the dose mass-dependence are lost. The energy could be a great option for dosing, (like it is in radiation therapies by Gy) but it has numerous drawbacks. These are discussed in this paper, trying to unify the dosing of ionizing and non-ionizing radiations.
\end{abstract}

\section{Keywords}

\section{Dosing, CEM, Specific-Absorption-Rate, Energy-Loss, Efficacy}

\section{Introduction}

Hyperthermia is an ancient medical intervention [1]. The fire (Sun) had symbolic significance in the ancient human cultures, so the heat delivery was naturally on top of the curative possibilities. The fight against cancer is even-aged with medicine itself, so it is not surprising that hyperthermia was applied for oncology, too. The ineffective heat deliveries were replaced with the modern electromagnetic heating techniques renewing medical hyperthermia. Oncological hyperthermia is an ideal combination therapy. It provides synergies with most of the conventional treatment modalities, boosts their efficacy and in many cases resensitizes the previously non-effective or even refractory treatments.

In contrary its long history, the state of oncological hyperthermia today is in its infancy. It has debates and questions about the present status of the method [2]. The main reason of this unprecedented long "infancy" is the missing of the dose to be applied in clinical practice [3].

Hyperthermia overheats the actual target. Temperature characterizes these methods. Due to the popular meaning of heating, the increased temperature became the measurement of the "dosing”. 
However, the temperature concept has numerous drawbacks:

1) Temperature is not a value which depends on the target's volume or mass as usually conventional doses do. Temperature of a heated object spreads automatically until the thermal equilibrium, and all the fractions of the target has the same amount, not like the "normal" doses having 50\% in the half volume.

2) Temperature is a consequence of the absorbed energy (specific absorption rate, SAR) which has the character of the dose (it is well quantitative and, depending on the technique, it could be well delivered). However, it has another factor: the living objects are usually good thermal conductors, promoting their thermal conductive exchange with various internal convective transports, as well as their homeostatic control tries to balance the unusual growth of the temperature in a part of whole. Consequently, the same SAR does not cause the same temperature rise in various targets [4], which does not satisfy the expectations of the temperature control.

3) The temperature (as general energy average) acts on all the energy-consuming processes. As the system is "immersed in the bath of energy", everything gets the action, irrespective if it is positive or negative in our process. For example, the physiological negative feedback is induced to compensate and control the disturbance, the locally high temperature. In consequence, unwanted supply of the tumour by fresh blood-delivered glucose and also the risk of further dissemination are created. The unselective energy delivery affects the healthy cells too, which could be extra toxicity limiting the dose of the heating.

4) Time-dosing by the actually reached temperature is introduced as dose concept: thermal isoeffective dose (TID) [5]. It usually refers to the effect at $43^{\circ} \mathrm{C}$, denoted by CEM $43^{\circ} \mathrm{C}$ (cumulative equivalent minutes; CEM).

\section{Dosing Methods}

It is not a question, the future of oncological hyperthermia depends on the definition of dosing prescribed as usual in medicine, [3]. This dose and the complete control are debated and cause the rare acceptance of hyperthermia in oncology. The actually applied doses are unsatisfactory [4] [6] [7], and due to the applied technologies it could not be significantly improved [8]. Oncothermia offers a new paradigm with nanoscopic heating to surmount this present complex problem.

CEM is proportional with the time of the exposure, and refers to $43^{\circ} \mathrm{C}$ where the necrotic cell-destruction is measured by Arrhenius fit in vitro [9]; noted by $\mathrm{CEM} 43^{\circ} \mathrm{C}$. This parameterization, however raises many doubts [10], and does not fit into the observed tumor-destruction [11].

These problems of characterization are accompanied by the non-uniform spatial distribution of the temperature, which of course results in the variation of the cell-destruction in different volumes of the tumor. This is "counted" with extra assumption of CEM $43^{\circ} \mathrm{CTx}$, which means how large volume of the full target reached the given $\mathrm{CEM} 43^{\circ} \mathrm{C}$ on average. Generally, $\mathrm{CEM} 43^{\circ} \mathrm{CT} 90$ is used $\left(90 \%\right.$ of the target reached the given $\mathrm{CEM} 43^{\circ} \mathrm{C}$ on average).

The doubts about the temperature-characterization are well-supported by the fact that the uniform and high temperature in the tumor by extreme whole body hyperthermia (WBH) has not results despite that its CEM value is $>15 \mathrm{CEM}^{\circ} 3^{\circ} \mathrm{CT} 100 /$ treatment. Note, the lower dose $\left(<5 \mathrm{CEM} 43^{\circ} \mathrm{CT} 90 /\right.$ treatment $)$ in local/regional heating and very heterogeneous temperature distribution offers better results.

Application of lower temperatures $\left(<40^{\circ} \mathrm{C}\right.$; mild-WBH) for treatments of longer periods ( $<2$ CEM $43^{\circ} \mathrm{CT} 100$ / treatment) showed surprisingly good efficacy, [12]. This application of fever-range activates the immune reactions and gives excellent addition to the tumor therapies [13]-[15], despite its low dose of CEM $43^{\circ} \mathrm{CT} 100 /$ treatment. This effect questions the necessity of the necrotic reference at $43^{\circ} \mathrm{C}$ in the time equivalence. So the $\mathrm{CEM} 43^{\circ} \mathrm{C}$ dose underestimates the value of the non-necrotic cell-killing under $43^{\circ} \mathrm{C}$.

The other side of the temperature range (high temperatures $>47^{\circ} \mathrm{C}$, like in ablation techniques) does not fit to the $\mathrm{CEM} 43^{\circ} \mathrm{C}$ dose concept either. This was demonstrated using in-vivo time-temperature [16] [17]. The $\mathrm{CEM} 43^{\circ} \mathrm{C}$ dose model over-estimates the accumulation of thermal damage for high-temperature thermal therapy applications.

Hyperthermia struggles the technical problems above, and sometimes it hinders the biological factors. The uncontrolled absorbed energy situation requests local control, the energy-intake which would be the natural dose measurement like in radiotherapy or like the chemical doses of medicine, cannot be applied here. Local, in-situ measurement is necessary to dose the treatment, and it can be the temperature only.

The dosing and control of the treatment is not only a technical and biological challenge. It is a hard problem of the medical application of hyperthermia. Without definite protocols it is a weak approach and has no possibil- 
ity for comparison of the results and also it does not give reliable possibility for the patients. However, the dose itself has raised numerous questions anywhere. The problem is mainly connected with the bio-variability which makes humans also individual. The dose has to be personalized, but then many points of the fixed protocol could not be fulfilled, as well as the collection of the cohorts for studies becomes complicated.

\section{Challenge of Energy-Dose}

The dose in electromagnetic radiations is well defined in case of ionization. The radiotherapy measures Gy $(\mathrm{J} / \mathrm{kg})$, as dose, which is a good quantitative parameter. However, its efficacy depends on many physiological and technical parameters (like the oxygenation of the tissue, the focusing arrangement of the devices, the fractionating possibilities, etc.). There are some surface burns representing the direct toxicity which can also limit the application. Anyway, the efficacy is measured by off-situ diagnosis (comparison of the before and after states), and the safety is fixed by the dose escalation studies, where severe toxicity blocks the further increase.

In case of non-ionizing radiation the situation changes: the absorbed energy specific absorption rate (SAR), which is measured like Gy in $\mathrm{J} / \mathrm{kg}$, is more and more time dependent in this case, because we need longer time to provide satisfactory energy to the target, and it decreases the possible energy of the incident beam due to burning of the surface. To provide enough energy in depth, it needs power of $>20 \mathrm{~W} / \mathrm{kg}$ [18], which is far over the surface tolerance. Usually intensive cooling of the surface is applied to avoid the blisters on the skin. This method also has various physiological feedback. When the cooling is too much, the feedback isolates the skin from the deeper cooling, reducing the blood-flow in the subcutane layer under the cooled area. This could increase the permittivity of the skin together with the decrease of its conductance by an intensified blood-perfusion [19].

Together with these, the deep-heating with low-frequency (non-ionizing, radiofrequency) needs bioelectromagnetic considerations, too. The intensified blood-perfusion increases the jump of the complex dielectric function at the incidence to the skin, which increases the jump of the electric field vector at the incidence. The increased potential drop will increase the risk of burn. The surface cooling is again complex and needs a definite balance to be optimal.

We have to consider that modern hyperthermia is always complementary and other methods have to be considered at hyperthermia applications. The lower limit of the hyperthermia dose is probably the normothermia, where nothing else has action but the complementary treatment. Slight heating locally or systemically has probably no effect directly on the tumor, but it helps to increase the immune effects, it enhances the complementary effects by the increased blood-flow and by the exponential temperature dependence of the chemical reactions (Arrhenius law). For the upper limit however, there are very definite technical and physiological parameters: the surface power-density of the signal is limited by the blistering at load above $0.5 \mathrm{~W} / \mathrm{cm}^{2}$ (60 min basis), the internal hot-spots could hurt the healthy tissue, and in the systemic application the physiology anyway limits at $42^{\circ} \mathrm{C}$.

The penetration depth of the non-ionizing radiation in biological material has strict frequency dispersion, quickly shortened by the growing frequency, and so contradicting with the demand of the high frequency for precise beaming. The energy-transmitters (boluses) have to be well-transferring of the applied electromagnetic effects without loss. Its other function is to limit the temperature of the body surface under the blistering threshold. The safety issues in actual cases usually modify the upper threshold of the action. Possible safe energyflow through the skin limits the Poynting vector (flow of energy in W/ $\mathrm{cm}^{2}$ ) [20], and in consequence the 60 min treatment has to have less than $0.5 \mathrm{~W} / \mathrm{cm}^{2}$ power density on the surface.

To avoid the overheating of the surface, intensive surface cooling is applied in most of the electromagnetic hyperthermia techniques. In this case the physiology has negative feedback control again. In hot environment the subcutaneous layers have vasodilatation, high blood-flow helps the heat-exchange with the environment, it radiates out the excess body-heat. In cold environment the blood-flow is limited, the surface layer isolates the body. Both cases change the heat- and electric-conductivity, as well as the dielectric properties of the skin layers. When the constrained forwarded power is applied, the voltage drop on the isolating (cooled) layer will be very high, a high voltage is necessary to pump through the requested constrain power. The relative high voltage lowers the current and less RF-current reaches the targeted deep-volumes. Consequently, the cooling process to avoid the burn has a positive feedback loop, the more cooling makes higher isolation, it induces higher voltage at the same incident power. The higher voltage makes more burning (electric burn using capillaries like "break- 
down”), so it requests more cooling which induces more electric burn and so on. Further consequence of the high cooling is that it lowers the RF-current which is the curative factor of the treatment.

Another further complication is the loss of a control parameter by the cooling process. The incident (controlled) energy loses its indefinite part by cooling and so the incident energy is not suitable anymore to characterize the process, the forwarded energy will massively differ from the absorbed energy by the target. This can be seen in the SAR measurements of the treatments.

The stationary rise of temperature could offer a possibility to calculate the SAR and the temperature in the heated living objects, [21] [22]. We know very well, that the heating energy makes temperature increase which depends on the mass $(m)$ of the heated object and its specific heat $(c)$ :

$$
\Delta Q=m c \Delta
$$

We know also, that the

$$
\Delta Q=P \Delta t
$$

where $P$ is the power and $\Delta t$ is the time duration when it was applied. So from the two equations:

$$
P \Delta t=m c \Delta
$$

and so the SAR (specific absorption rate) is:

$$
\operatorname{SAR}\left[\frac{\mathrm{W}}{\mathrm{kg}}\right]=\frac{P}{m}=c \frac{\Delta T}{\Delta t}\left[\frac{\mathrm{C}^{\mathrm{o}}}{\mathrm{s}}\right]
$$

When phantoms are heated (no inhomogeneity, no physiologic feedback [blood-flow]) the $\frac{\Delta T}{\Delta t}$ is linear.

Using the units which are common in practical physiology:

$$
\mathrm{SAR}[\mathrm{W} / \mathrm{kg}]=\left.\left.\frac{4182}{60} \cdot \frac{\Delta T}{\Delta t}\right|_{t=0}\left[\mathrm{C}^{\circ} / \mathrm{min}\right] \cong 70 \cdot \frac{\Delta T}{\Delta t}\right|_{t=0}\left[\mathrm{C}^{\circ} / \mathrm{min}\right]
$$

Consequently, SAR is calculable from the starting time-derivative of the temperature. This is the so-called "adiabatic SAR", because the interactions with the environment are neglected, (Figure 1).

The phantom experiments are static. There is no blood-flow feedback and the phantom is homogeny.

You can see the linearity from the precise professional validation experiment [23], (8 MHz; Thermotron RF-8, Yamamoto Vinita, Japan), measured the SAR using agar phantom (Figure 2).

So the actual SAR values in depth 1.8, 3.5, 7 and $12.2 \mathrm{~cm}$ are $90,70,40,35 \mathrm{~W} / \mathrm{kg}$, respectively. The average SAR (overestimated) is

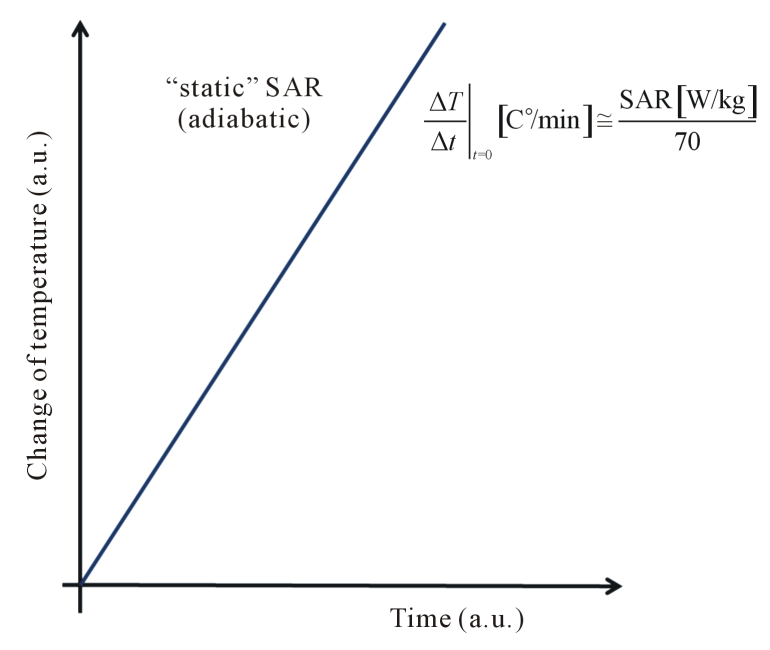

Figure 1. Heating a homogeneous phantom shows "static" SAR, dynamic feedback does not exist. 


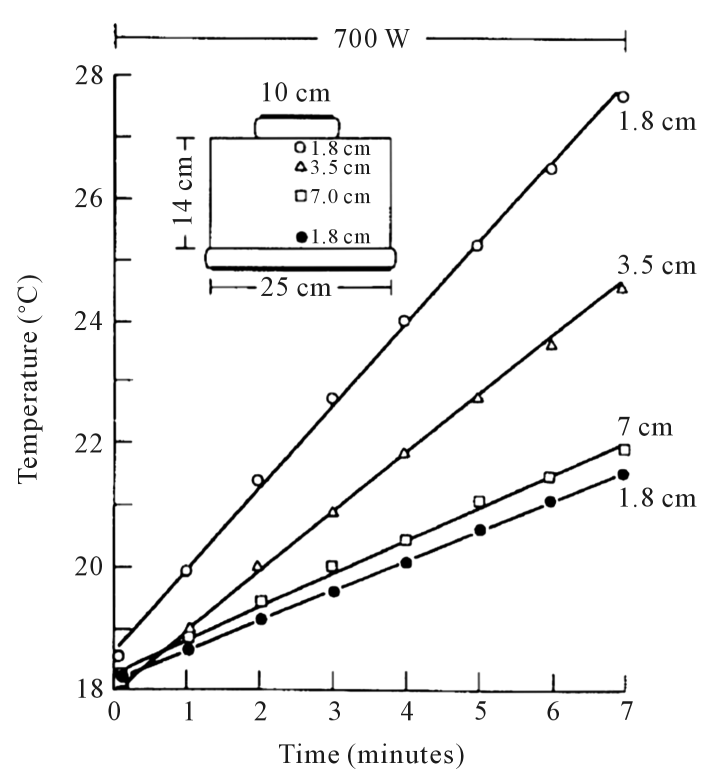

Figure 2. The measured temperature development by Thermotron. The sizes of the agar phantom are in the insert [23].

$$
\mathrm{SAR}_{\text {average }}=70 \frac{\mathrm{W}}{\mathrm{kg}}
$$

The phantom (on the insert) volume is easy to be calculated $\approx 1.4$ liter, which using the agar density [24] is 1.4 $\mathrm{kg}$ mass. So the absorbed power in this entire phantom is $70 \times 1.4=98 \mathrm{~W}$.

The applied power for heating was $700 \mathrm{~W}$ (written in the paper and on the figure as well). The heating energy-efficacy is $14 \%$. This means they are not able to use the absorbed energy to measure the dose; due to the low efficacy we have idea only by the temperature about how much energy could be absorbed.

Radiative hyperthermia (120 MHz; phase array, BSD2000, BSD Medical USA) used beef-phantom in SigmaEye-60, [25]. The measurement was performed in a high level lab of MIT, [26]. The temperature development is shown in Figure 3.

The mass of the phantom-beef on Sigma-Eye was $10.9 \mathrm{~kg}$. Four channels were applied around the phantom (in crossing) to heat, each channel had $215 \mathrm{~W}$ power, so the complete power applied was $860 \mathrm{~W}$. The absorbed heat energy in beef (see Figure 3) was $7.44 \mathrm{~W} / \mathrm{kg} \times 10.9 \mathrm{~kg}=81 \mathrm{~W}$. In this case the energy-efficacy of heating is $9.4 \%$. Again, the complete power in the phantom was less than $100 \mathrm{~W}$, and due to the low efficacy only the temperature measurement could carry information about the energy absorption.

Another capacitive device (13.56 MHz; ThermMed, LLC, USA), (Figure 4) applied power of $600 \mathrm{~W}$ for 10 min, [27]. The gain of temperature is $16.5^{\circ} \mathrm{C}$, which gives $\approx 115.5 \mathrm{~W} / \mathrm{kg}$ SAR. This enormous SAR heats a SCID nude mice with a complete mass of $\sim 10 \mathrm{~g}$. The entire mice were loaded byapprox. $2.3 \mathrm{~W}$, while the tumor ( $>1 \mathrm{~g}$ ) had approx. $0.116 \mathrm{~W}$. The actual efficacy of the energy-use is approx. $0.5 \%$.

It is obvious that the energy cannot provide dose with this efficacy.

Other capacitive device (13.56 MHz; EHY2000+, Oncotherm, Germany) phantom measurement was measured on pork-meat phantom, [28] (Figure 5).

The applied power was $100 \mathrm{~W}, 10 \mathrm{~cm}$ diameter electrode was applied, chopped pork-meat as phantom was $2.3 \mathrm{~kg}$ in mass. The SAR values of $6,24 \mathrm{~cm}$ depth are $50 \mathrm{~W} / \mathrm{kg}$ and $36 \mathrm{~W} / \mathrm{kg}$. The average of the SAR absorption is around $40 \mathrm{~W} / \mathrm{kg}$ (definitely more than $36 \mathrm{~W} / \mathrm{kg}$ ). The absorbed energy is $2.3 \mathrm{~kg} \times 40 \mathrm{~W} / \mathrm{kg}=92 \mathrm{~W}$, so the efficacy is $92 \%$.

The energy-efficacy is $92 \%$. This could be the basis of the energy-dose measurement.

The above phantom measurements well show why only the temperature remains to measure the dose in most of the cases, the energy has lost its characteristic value. Furthermore, the real, effective absorbed energy in the phantoms were not as high as expected, in these cases it did not exceed the $100 \mathrm{~W}$. 


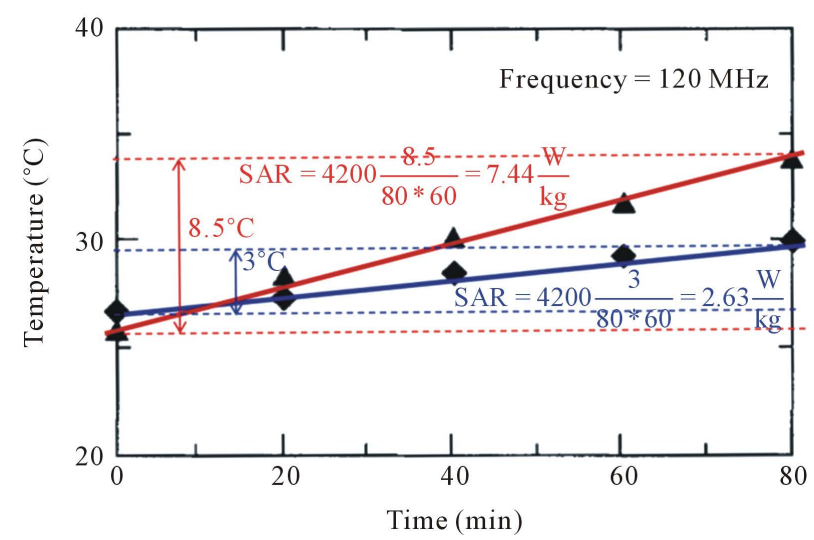

Figure 3. The best SAR is $7.44 \mathrm{~W} / \mathrm{kg}$ at $120 \mathrm{MHz}$ frequency for beef-phantom [25].

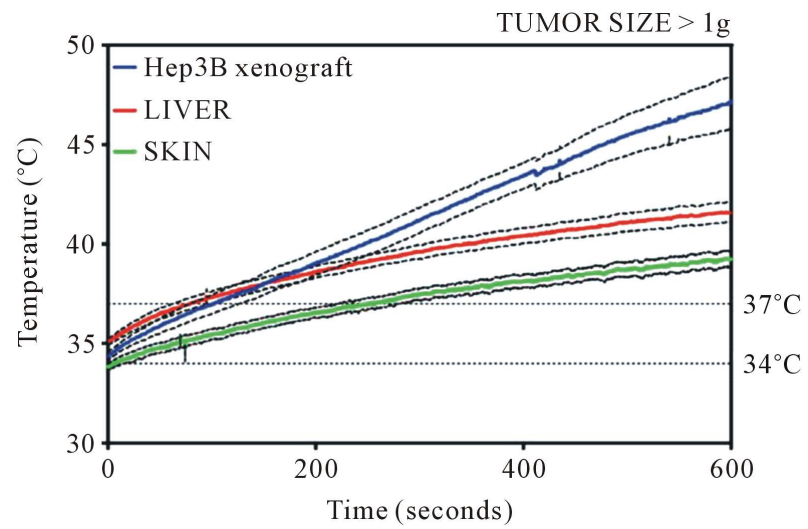

Figure 4. Heating nude mice with capacitive device (13.56 MHz; ThermMed, LLC, USA), tumor size is $>1 \mathrm{~g}$, [27].

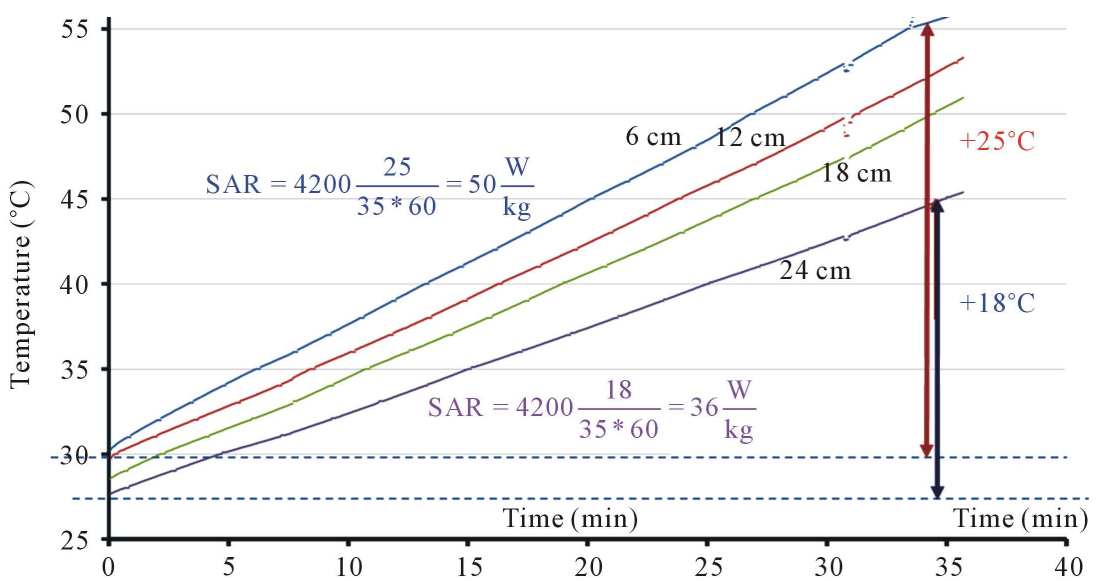

Figure 5. Meat (chopped pork) phantom measurement (13.56 MHz, EHY2000+, Oncotherm, Germany, [28].

When the measurement is not performed on phantom, real physiologic feedback could be expected. The Pennes equation of heat transfer describes this situation:

$$
\rho c \frac{\partial T}{\partial t}+c_{b} \rho_{b} w_{b}(T)\left(T-T_{b}\right)=\lambda \nabla^{2} T+\mathrm{SAR} \cdot S(T)+q_{0} 1.1^{(T-36.6)}
$$


where $\rho$-mass-density of the tissue, $c$-specific heat of the tissue, $T$-temperature of the tissue, $t$ - time for heating, $c b, \rho b, w b$, and $T b$ are the specific heat, density, flow-rate and the temperature of the blood, respectively. The $\lambda$ is the heat-diffusion constant, SAR is the value of specific absorption rate, while $q_{0}$ characterizes the basal metabolic rate, $S(T)$ is the gain of SAR (SARgain) due to the change of the temperature. The body temperature in rest is assumed $36.6^{\circ} \mathrm{C}$.

In stationary case the $\frac{\partial T}{\partial t}=0$, and $\nabla^{2} T=0$. We neglect the changes of $S(T)$ and the metabolic rate by temperature, too. In this case:

$$
\Delta T=\left(T-T_{b}\right)=\frac{\mathrm{SAR}}{c_{b} \rho_{b} w_{b}(T)}
$$

Here, the blood perfusion rate is given in the SI system, that is in: $\frac{\mathrm{m}^{3}}{\mathrm{sec}^{3}}$. In the physiologic equation, this appears in physiologic units, that is: $\frac{\mathrm{ml}}{\min \cdot 100 \mathrm{~g}}$. To converse between these two:

$$
w_{b}(T)=\frac{10^{-6}}{6} w_{b p h}(T)
$$

Putting this into the previous equation, we get:

$$
\Delta T=\frac{\mathrm{SAR}}{c_{b} \rho_{b} w_{b}(T)}=\frac{\mathrm{SAR}}{1.1 \times 10^{3} \times 4.5 \times 10^{3} \times \frac{10^{-6}}{6} w_{b p h}(T)}=1.3 \frac{\mathrm{SAR}}{w_{b p h}(T)}
$$

Note, the result in [23] has a little higher converting factor:

$$
\Delta T=1.4 \frac{\mathrm{SAR}}{w_{b p h}}
$$

probably using different basic data from the literature (we used data from [29]), (see Figure 6).

The calculation shows that the dynamic equilibrium is definitely also SAR-dependent, and no way to determine it when the technical conditions (the energy-efficacy) do not allow a good approximation of energy.

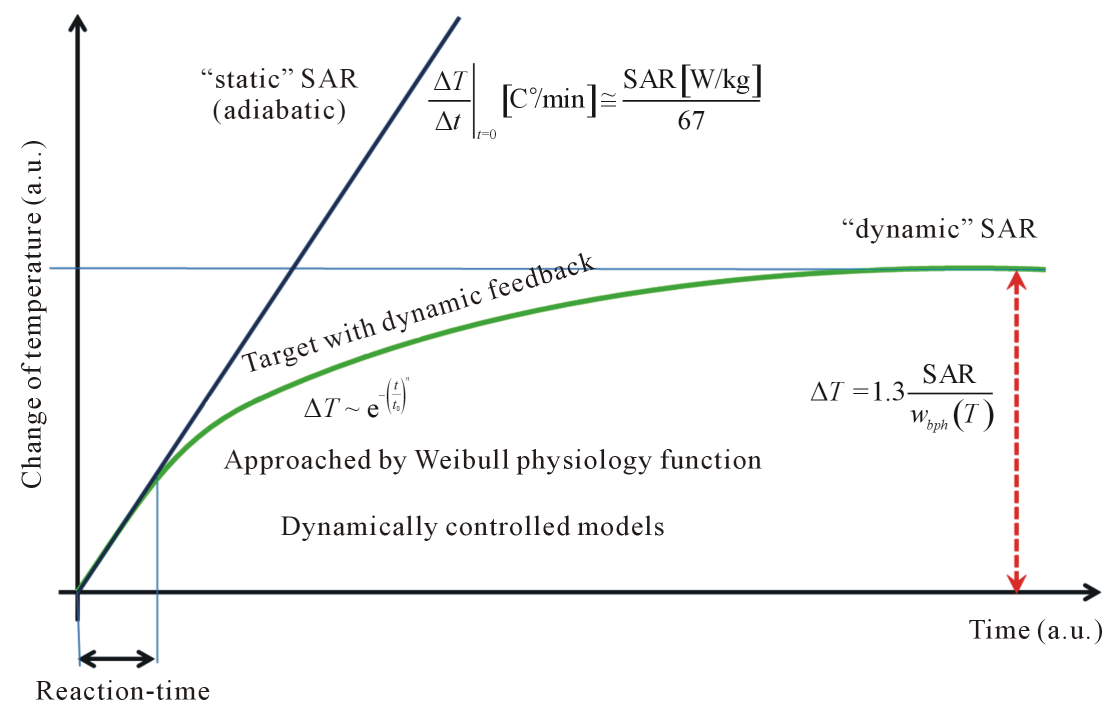

Figure 6. Difference between the static and dynamic heating condition is basically caused by the negative feedback of blood-flow, which equalizes the temperature and keeps its stationery in a given volume (which changes by time of course), [25]. 


\section{Conclusion}

The main block to use the specific absorption rate (specific absorbed energy) in case of non-ionizing radiation is mainly a technical limit, due to the low efficacy of the energy-transfer. The temperature could be the dose in those cases when there is no other possibility to control (or at least having info) the absorbed energy.

\section{References}

[1] Seegenschmiedt, M.H. and Vernon, C.C. (1996) A Historical Perspective on Hyperthermia in Oncology. In: Seegenschmiedt, M.H., Fessenden, P. and Vernon, C.C., Eds., Thermo-Radiotherapy and Thermo-Chemiotherapy, Springer, Berlin, 3-46. http://dx.doi.org/10.1007/978-3-642-60938-1 1

[2] Baronzio, G., Jackson, M., Lee, D. and Szasz, A. (2013) Editorial of the Conference of the International Clinical Hyperthermia Society 2012. Conference Papers in Medicine, 2013, Article ID: 690739.

[3] Jones, E., Thrall, D., Dewhirst, M.W. and Vujaskovic, Z. (2006) Prospective Thermal Dosimetry: The Key to Hyperthermia's Future. International Journal of Hyperthermia, 22, 247-253. http://dx.doi.org/10.1080/02656730600765072

[4] van der Zee, J. (2005) Renewing Oncological Hyperthermia-Oncothermia. Presentation in Mumbay, India. http://www.oalib.com/references/7745442

[5] He, X., Bhowmick, S., and Bischof, J.C. (2009) Thermal Therapy in Urologic Systems: A Comparison of Arrhenius and Thermal Isoeffective Dose (TID) Models in Predicting Hyperthermic Injury. Journal of Biomechanical Engineering-Transactions of the ASME, 131, Article ID: 074507.

[6] Vincze, Gy. and Szasz, A. (2006) Dose Concept of Oncological Hyperthermia: Heat-Equation Considerint the Cell Destruction. Journal of Cancer Research Therapeutics, 2, 171-181. http://dx.doi.org/10.4103/0973-1482.29827

[7] Szasz, A. (2007) Hyperthermia, a Modality in the Wings. Journal of Cancer Research and Therapeutics, 3, 56-66. http://dx.doi.org/10.4103/0973-1482.31976

[8] Szasz, A. (2013) “Quovadis” Oncologic Hyperthermia? Hindawi Publishing, Corporation Conference Papers in Medicine, 2013, Article ID: 201671.

[9] Sapareto, S.A. and Dewey, W.C. (1984) Thermal Dose Determination in Cancer Therapy. International Journal of Radiation Oncology-Biology, Physics, 10, 787-800. http://dx.doi.org/10.1016/0360-3016(84)90379-1

[10] Wust, P., Hildebrandt, B., Sreenivasa, G., Rau, B., Gellermann, J., Riess, H., Felix, R. and Schlag, P.M. (2002) Hyperthermia in Combined Treatment of Cancer. Lancet Oncology, 8, 487-497. http://dx.doi.org/10.1016/S1470-2045(02)00818-5

[11] de Bruijne, M., van der Holt, B., van Rhoon, G.C. and van der Zee, J. (2010) Evaluation of CEM43 ${ }^{\circ}$ T90 Thermal Dose in Superficial Hyperthermia, A Retrospective Analysis. Strahlentherapie Onkologie, 186, 436-443. http://dx.doi.org/10.1007/s00066-010-2146-X

[12] Kraybill, W. and Olenki, T. (2002) A Phase I Study of Fever-Range Whole Body Hyperthermia (FR-WBH) in Patients with Advanced Solid Tumors: Correlation with Mouse Models. International Journal of Hyperthermia, 18, $253-266$. http://dx.doi.org/10.1080/02656730110116704

[13] Toyota, N., Strebel, F.R., Stephens, L.C., Matsuda, H. and Bull, J.M. (1997) Long Duration—Mild Whole Body Hyperthermia with Cisplatin: Tumour Response and Kinetics of Apoptosis and Necrosis in a Metastatic at Mammary Adenocarcinoma. International Journal of Hyperthermia, 13, 497-506. http://dx.doi.org/10.3109/02656739709023548

[14] Sakaguchi, Y., Makino, M., Kaneko, T., et al. (1994) Therapeutic Efficacy of Long Duration-Low Temperature Whole Body Hyperthermia When Combined with Tumor Necrosis Factor and Carboplatin in Rats. Cancer Research, 54, 2223-2227.

[15] Ostberg, R. (2000) Use of Mild, Whole Body Hyperthermia in Cancer Therapy. Immunological Investigations, 29, 139-142. http://dx.doi.org/10.3109/08820130009062297

[16] Moritz, A.R. and Henriques, F.C. (1947) Studies of Thermal Injury. II. The Relative Importance of Time and Surface Temperature in the Causation of Cutaneous Burns. American Journal of Pathology, 23, 695-720.

[17] Henriques, F.C. (1947) Studies of Thermal Injury V. The Predictability and the Significance of Thermally Induced Rate Processes Leading to Irreversible Epidermal Injury. Archives of Pathology, 43, 489-502.

[18] Wust, P. (2005) Thermoregulation in Humans-Experiences from Thermotherapy. Conference in Stuttgart.

[19] Vuksanovic, V., Sheppard, L.W. and Stefanovska, A. (2008) Nonlinear Relationship between Level of Blood Flow and Skin Temperature for Different Dynamics of Temperature Change. Biophysical Journal: Biophysical Letters, L78-L80.

[20] Stoll, A.M. (1967) Heat Transfer in Biotechnology. In: Hartnett, J.P. and Irvine, T.F., Eds., Advances in Heat Transfer, Academic Press Inc., New York and London, 65-139. http://dx.doi.org/10.1016/s0065-2717(08)70273-9

[21] Paulides, M.M., Bakker, J.F. and van Rhoon, G.C. (2007) An Electromagnetic Head and Neck Hyperthermia Applica- 
tor: Experimental Phantom Verification and FDTD Model. International Journal of Radiation Oncology*Biology* Physics, 68, 612-620. http://dx.doi.org/10.1016/j.ijrobp.2007.01.035

[22] Wust, P. (2005) Thermoregulation in Humans, Experiences from Thermotherapy. Proceedings of the Workshop on Subtle Thermal Effects of RF-Fields in Vitro and in Vivo, Stuttgart, 21-23 November 2005.

[23] Song, C.W., Rhee, J.G., Lee, C.K.K. and Levitt, S.H. () Capacitive Heating of Phantom and Human Tumors with an 8 MHz Radiofrequency Applicator (Thermotron rf-8). International Journal of Radiation Oncology*Biology* Physics, 12, 365-372. http://dx.doi.org/10.1016/0360-3016(86)90352-4

[24] Aranda-Lara, L., Torres-García, E. and Oros-Pantoja, R. (2014) Biological Tissue Modeling with Agar Gel Phantom for Radiation Dosimetry of 99mTc. Open Journal of Radiology, 4, 44-52. http://dx.doi.org/10.4236/ojrad.2014.41006

[25] Fenn, A.J. and King, G.A. (1994) Adaptive Radiofrequency Hyperthermia-Phased Array System for Improved Cancer Therapy: Phantom Target Measurements. International Journal of Hyperthermia, 10, 189-208. http://dx.doi.org/10.3109/02656739409009343

[26] Fenn, A.J. and King, G.A. (1999) Adaptive Radiofrequency Hyperthermia-Phased Array System for Improved Cancer Therapy: Phantom Target Measurements. MIT Lincoln Laboratory Technical Report 999, 19 November 1993.

[27] Raoof, M., Cisneros, B.T., Corr, S.J., Palalon, F., Curley, S.A. and Koshkina, N.V. (2013) Tumor Selective Hyperthermia Induced by Short-Wave Capacitively-Coupled RF Electric-Fields. PLoS ONE, 8, e68506.

[28] Nagy, G., Meggyeshazi, N. and Szasz, O. (2013) Deep Temperature Measurements in Oncothermia Processes. Conference Papers in Medicine, 2013, Article ID: 685264. http://dx.doi.org/10.1155/2013/685264

[29] Erdmann, B., Lang, J. and Seebass, M. (1998) Optimization of Temperature Distributions for Regional Hyperthermia Based on a Nonlinear Heat Transfer Model. Annals of NYAS, 858, 36-46. 